\title{
The two directions of cancer nanomedicine
}

\author{
The cancer nanomedicine field is heading in two directions - debating whether the clinical translation of \\ nanomaterials should be accelerated or whether some of the long-standing drug delivery paradigms have to be \\ challenged first.
}

A t the International Conference on Nanomedicine and Nanobiotechnology that was held in Munich, 16-18 October, the most striking talk was not given by a scientist, nor a clinician, but by Lora Kelly - a six-year pancreatic cancer survivor. By telling her story of how it actually feels to receive chemotherapy, immunotherapy and radiation, she reminded everyone about the urgent need to improve cancer treatment regimes. The main goal remains to kill the cancer; however, it has become more evident how equally important it is to improve the quality of life of patients during treatment, that is, to reduce the often devastating side effects. This is where nanomedicine comes in.

Nanomaterials have the potential to direct drugs to specific tissues and to improve drug activity, as well as its transport in blood. Indeed, nanoparticles could ensure that therapeutic treatments act locally and not systemically, and thus improve anticancer efficacy while reducing damage to healthy tissues. However, recent setbacks, including the bankruptcy of a prominent nanomedicine company ${ }^{1}$ and the less than $1 \%$ delivery efficiency claim ${ }^{2}$ (quoted at every cancer nanomedicine conference on at least one slide) have stirred discussions about the usefulness of nanomedicines for cancer treatment. Some argue that the field is stuck in preclinical animal models owing to a lack of insight into the basics of nanomaterial-tissue interactions in the human body, from traversing biological barriers to clearance.

While less than $1 \%$ delivery efficiency might not be much, pharmacological parameters, such as peak drug concentration, clearance rate and elimination half-life, are often not as bad ${ }^{3}$, and these should be considered with equal importance. Moreover, there are also clinical success stories of nanomedicines. Onpattro, a lipid nanoparticle-based short interfering RNA (siRNA) drug for the treatment of polyneuropathies, was approved by the US Food and Drug Administration in 2018, marking the first approved nanoparticle for nucleic acid delivery. In a Comment in this issue, Akinc et al. report the endeavour of developing this nanomedicine, from the idea to preclinical and clinical testing ${ }^{4}$,

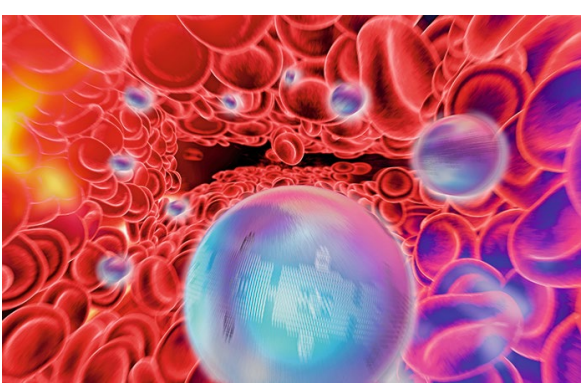

Credit: Science Photo Library - PASIEKA/Brand X Pictures/Getty

to the final approval. There are further many opportunities for nanomaterials complementary to drug delivery, including bioimaging, modulation of the immune system and the tumour microenvironment, and, of course, local administration.

From an Editorial perspective, the ongoing discussion is reflected in the many manuscripts we receive, which often include both basic investigations and claims of clinical application. Naturally, this can lead to mixed peer-review reports echoing the disconnection between clinical vision and fundamental science. Reviewers with a background in materials science or biomedical engineering often point out the gaps in the basic understanding of how a nanomaterial interacts with the biological environment, and clinicians would like to see more preclinical animal work. Indeed, a thorough fundamental study does not always need the claim of a specific application, as it might be exactly such overstatements that have precluded the field to deliver on the promise of revolutionizing drug delivery. Along the same line, studies of nanoparticle transport through specific cells or nanomaterial-cell interactions at a molecular scale, do not necessarily require complex in vivo models; by contrast, applied studies claiming a therapeutic benefit need a robust in vivo validation in a relevant animal model - preferably with an intact immune system.

Going back to the goal of improving a patient's life, possible side effects and impact on tissues other than tumours should also be reported. However, this data is often found, at best, somewhere in the supplementary information. Regardless of the mouse model, the discussion rarely goes beyond the weight loss and the histology of organs. If the idea is to improve therapies, side effects need to be thoroughly investigated - even at an early preclinical stage. Similarly, we will make sure that studies claiming superiority of a therapeutic treatment compared to state-of-the-art treatment regimes are reviewed by clinical experts to ensure that clinical translation is - at least - possible and feasible. Also, keeping regulatory requirements in mind, the more complex the new nanoparticle or nanoscale delivery agent, the more difficult it will be to get approval; and this is a valid criticism.

At Nature Nanotechnology, we consider both clinically relevant manuscripts and fundamental studies investigating the various barriers nanoparticles face on their journey through the body. We endeavour to assess the manuscripts we receive as fairly and consistently as possible, with the ongoing discussion in mind. We look forward to learning about possible alternative mechanisms and the heterogeneity of the enhanced permeability and retention (EPR) effect, nanoparticle interactions in the liver, spleen and kidneys during clearance, migration of nanomaterials through the tumour microenvironment, and nanoparticle uptake, lysosomal escape (or not) and transport in different cell types. Such studies will shine a light on nanomaterial-tissue interactions, and also greatly contribute to the development of improved nanomedicines. Equally important, detailed investigations of nanoparticles in preclinical animal models as well as relevant organoid cultures will allow the optimization of treatment strategies and the reduction of side effects. Regardless of the aim, we urge authors to calibrate their claims in accordance with their data and scope of the investigation to preserve trust in cancer nanomedicine as a whole.

Published online: 4 December 2019 https://doi.org/10.1038/s41565-019-0597-5

References

1. Ledford, H. Nature 533, 304-305 (2016).

2. Wilhelm, S. et al. Nat. Rev. Mater. 1, 16014 (2016).

3. McNeil, S. E. Nat. Rev. Mater. 1, 16073 (2016).

4. Akinc, A. et al. Nat. Nanotechnol. https://doi.org/10.1038/s41565 019-0591-y (2019). 